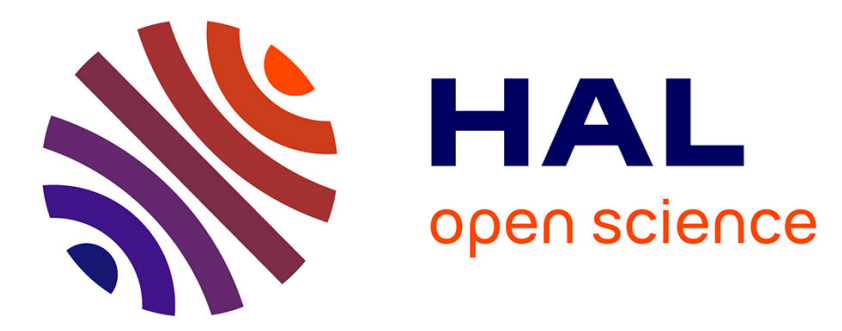

\title{
From local growth to global optimization in insect built networks
}

\author{
Andrea Perna, Pascale Kuntz, Guy Theraulaz, Christian Jost
}

\section{To cite this version:}

Andrea Perna, Pascale Kuntz, Guy Theraulaz, Christian Jost. From local growth to global optimization in insect built networks. P. Lio and D. Verma. Biologically Inspired Networking and Sensing: Algorithms and Architectures, IGI Global, pp.132-144, 2011, 10.4018/978-1-61350-092-7.ch007 . hal00600664

\section{HAL Id: hal-00600664 https://hal.science/hal-00600664}

Submitted on 15 Jun 2021

HAL is a multi-disciplinary open access archive for the deposit and dissemination of scientific research documents, whether they are published or not. The documents may come from teaching and research institutions in France or abroad, or from public or private research centers.
L'archive ouverte pluridisciplinaire HAL, est destinée au dépôt et à la diffusion de documents scientifiques de niveau recherche, publiés ou non, émanant des établissements d'enseignement et de recherche français ou étrangers, des laboratoires publics ou privés. 


\title{
From Local Growth to Global Optimization in Insect Built Networks
}

\author{
Andrea Perna \\ Complex Systems Institute of Paris, France \& Uppsala University, Sweden \\ Pascale Kuntz \\ Site Ecole Polytechnique de l'Université de Nantes, France \\ Guy Theraulaz \\ Université de Toulouse, France \& CNRS, France \\ Christian Jost \\ Université de Toulouse, France \& CNRS, France
}

\begin{abstract}
Social insect colonies build large net-like systems: gallery and trail networks. Many such networks appear to show near-optimal performance. Focusing on the network system inside termite nests we address the question how simple agents with probabilistic behaviour can control and optimize the growth of a structure with size several magnitude orders above their perceptual range. We identify two major classes of mechanisms: (i) purely local mechanisms, which involve the arrangement of simple motifs according to predetermined rules of behaviour and (ii) local estimation of global quantities, where sizes, lengths, and numbers are estimated from densities, concentrations, and traffic. Theoretical considerations suggest that purely local mechanisms work better during early network formation and are less likely to fall into local optima. On the contrary, estimation of global properties is only possible on functional networks and is more likely to work through pruning. This latter mechanism may contribute to restore network functionalities following unpredicted changes of external conditions or network topology. An analysis of the network properties of Cubitermes termite nests supports the role of both classes of mechanisms, possibly in interplay with environmental conditions acting as a template.
\end{abstract}




\section{ARE INSECT-MADE NET-LIKE STRUCTURES OPTIMAL?}

The nests of social insects are among the most impressive objects built by animals, and this for several reasons. First, they can be extremely big: up to several magnitude orders bigger than insects themselves. Second, they usually present a coherent and harmonious global organization even at the larger scale. Third, they are not produced by extremely intelligent animals, but by tiny insects with somewhat noisy, seemingly unpredictable behaviour. These properties make insect nests particularly interesting in a perspective of bioinspiration.

If these structures optimize some functionality, then we can imagine mimicking insect behaviour to build efficient artificial systems that accomplish similar functionalities.

How do the insects come to build such complex structures?

The question allows for two different interpretations: the first focuses on the evolutionary history of insects while the second focuses on the building mechanisms:

1. By what evolutionary processes social insects have acquired the capability of building complex structures?

2. What building mechanisms and actions at the individual level lead to the formation of the global structure?

Let us illustrate the two interpretations with an example dealing with nest building, if not directly with network like structures. In an emblematic chapter of "The Origin of Species" Charles Darwin (1859, chapter 6 ) thinks about honeybee combs and states that:

He must be a dull man who can examine the exquisite structure of a comb, so beautifully adapted to its end, without enthusiastic admiration.
We hear from mathematicians that bees have practically solved a recondite problem, and have made their cells of the proper shape to hold the greatest possible amount of honey, with the least possible consumption of precious wax in their construction. (...) it seems at first quite inconceivable how they can make al $l$ the necessary angles and planes, or even perceive when they are correctly made.

Darwin's explanation is in terms of natural selection: in the same chapter he argues that "cells constructed like those of the bee or the wasp gain in strength, and save much in labour and space". It is natural that the instincts of bees must have undergone "numerous, successive, slight modifications" that led to the construction of more and more efficient structures (Darwin, 1859).

An alternative discussion of the very same phenomenon, but this time focusing on building mechanisms is found in D'Arcy Thompson's “On Growth and Form" (Thompson, 1992):

the direct effort of the wasp or bee may be supposed to be limited (...) to the making of little hemispherical cups, as thin as the nature of the material permits, and packing these little round cups as close as possible together. It is then conceivable, and indeed probable, that the symmetrical tensions of the semi-fluid films should suffice (however retarded by viscosity) to bring the whole system into equilibrium, that is to say into the configuration which the comb actually assumes.

For Darwin, bees make combs with minimal surface-volume ratio because this configuration confers the maximum selective advantage; for D'Arcy Thompson, the minimal surfaces appear because this is the configuration naturally assumed by semi-fluid films, be they soap-bubbles, cells of a segmenting egg or honey combs.

In principle there is no contradiction between the two explanations: bees could benefit from having cells with minimum surface to volume ratio 
and also get this ratio minimized almost for free because this is the minimal energetic configuration. However, these examples illustrate well how difficult is assessing the optimality of insect built structures: when we address the question about evolutionary processes, the surface-volume ratio of honey combs is the objective of optimization, but when we focus on the building process the same minimal surface-volume ratio can be an epiphenomenon of the building mechanism, with no adaptive value. (Incidentally, let us mention that Thompson's explanation is not unique in its kind and a similar "mechanical" explanation had been put forward almost two centuries earlier by Buffon (1753). For Buffon the motor of surface minimization is not the tension of the wax, but the pressure exerted by the body of bees inside the cells.Amore recent paper supports the mechanism proposed by D'Arcy Thompson for honeybee combs (Pirk et al., 2004). However, let us say that even if the origin of the hexagonal cells is in physical forces and not in the behaviour of bees, this does not rule out the possibly important role of natural selection. For instance, was could have been selected as a building material because its melting point and viscous properties easily produce the hexagonal pattern).

An additional point that comes out from these examples is that words such as "optimal" and "efficient" do not have the same meaning in biology and in computer science. In biology, the concept of optimality is intrinsically related to the concept of biological fitness (roughly, the ability of an individual to propagate its genes). In other words, it is not sufficient that a biological object maximizes or minimizes a particular function, but the function optimized must also confer a selective advantage to the individuals. For Darwin it is not sufficient that bees build cells with minimal surfaces, it is important that they save "labour" and "costly wax": surface minimization must allow them to save energy that can be reinvested in producing and nourishing a larger offspring.
In practice, the only means to assess the biological efficiency of insect nests would be to measure the reproductive success of the colonies inhabiting them and relate it to measures of nest size, shape and organization, which clearly is extremely difficult. Indeed, most studies of biological optimality do not aim at finding optimality in a biological system, but take the assumption that the system is "optimal" as a starting point to address questions about the constraints and the objectives that have shaped its actual properties (Parker \& Smith, 1990).

In the rest of this paper we will not use words such as "efficient" and "optimal" in their biological meaning, but in the sense they usually assume in mathematics and computer science, that is, to indicate how close the solution found by insects is to the optimization of a particular function, without necessarily implying a selective advantage in the biological sense.

In this case, some net-like structures built by insects were shown to optimize different functionalities. In particular, the foraging systems of ants (Acosta et al, 1993; Solé et al., 2000; Buhl et al. 2009) and termites (Lee et al., 2007) tend to maximize food intake for a given total length of the transportation network (galleries and trails) required to collect it. Ant galleries also form efficient transportation networks in terms of distances between destinations and robustness (Buhl et al., 2004a).

In this paper, we focus on the analysis of the gallery system in termite nests. For some species, this complex system forms a 3D network which can be described by a graph $\mathrm{G}=(\mathrm{V}, \mathrm{E})$ : the vertices V represent the chambers and the edges E represent the connections between the chambers (fig. 1).

We have recently shown that the topological structure of the connections in specimens of the genus Cubitermes is particularly adapted to fulfil specific functions such as communication efficiency and ease of defence (Perna et al., 2008; Perna et al., 2008a) 
Figure 1. A. A Cubitermes nest. The nest is $\sim 30 \mathrm{~cm}$ high and has the typical mushroom shaped appearance. B. Virtual cast of the same nest. C. Virtual nest cut to show the internal chambers and galleries. Chambers are mapped to network vertices, galleries to edges. D. Detail of the nest, in a similar representation as in B, but here the structure has been thinned to render the paths of interconnections visible. One such path is marked by red dots. E. Graph representation of the same nest. Vertex colours reflect the layer where they are in the nest.
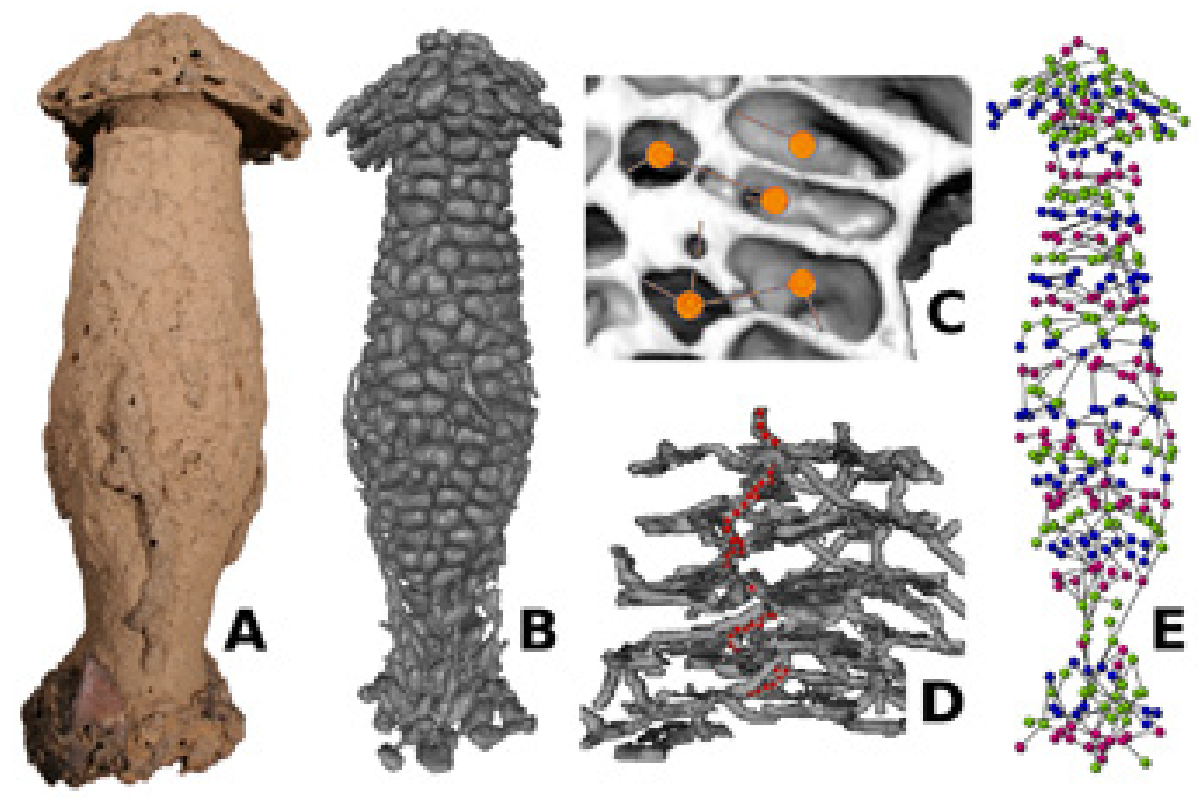

Here, we first complete some results on the communication efficiency: we show that this latter is far better than the one reached in random networks of similar sizes and that this property may be partly explained by the presence of very particular 3D sub-graphs (like "ramps"). The second part of the paper addresses the question of the building mechanisms of such complex structures: how do the insects control and regulate the growth of a structure that is so much bigger than their perception range? In other words, how can global optimization result from local growth rules? We here distinguish two families of processes: (i) local rules which involve the arrangement of very simple motifs, but result in globally efficient structures, and (ii) local estimation of global properties which allows the agents to regulate their own behaviour. We show how these two processes can be involved in the formation of the gallery networks in the termite nests.

\section{Optimization of Global Properties}

In order to get quantitative measures of global nest properties, we need a convenient representation for the complex forms of insect nests and trails. Such a representation should describe both the small scale (the one more likely accessible to the perception and action range of insects) and the large scale (the whole structure with the properties it optimizes). This requirement is necessary if we want to explore the relationship between the two scales of representation. For the analysis of termite nests, graphs are particularly well suited. Generally speaking, they are characterized by several measures of the local organization (vertex degree, vertex properties, assortativity or disassortativity between vertices, clustering coefficient etc.), as well as the intermediate (frequency of specific motifs, presence of cycles) and the large scale properties (diameter, average path length, distri- 
bution of betweenness and closeness centrality etc.; Boccaletti et al., 2006), as seen in Figure 1.

The termite nests analyzed in this paper have been built by African termites of the genus Cubitermes. The nests were imaged with computer tomography and the internal transportation network was extracted with image analysis techniques. In this network, a vertex vi in $\mathrm{V}$ represents a physical chamber and an edge $e i j=\{v i, v j\}$ in E depicts a physical gallery between chambers $v i$ and $v j$.

The efficiency to navigate the network from vertex to vertex is well quantified by its "global topological efficiency"(Latora \& Marchiori, 2001), which for a network with $\mathrm{N}$ vertices is given by the following equation:

$$
L=\frac{1}{N(N-1)} \sum_{\forall v_{i}, v_{j}} \frac{1}{d\left(v_{i}, v_{j}\right)}
$$

where $d(v i, v j)$ corresponds to the shortest topological path between the vertices $v i$ and $v j$.
This quantity is bounded in the interval $[0,1]$ and is higher for networks where most pairs of vertices are connected with short paths.

Figure 2 reports the average efficiency for five termite nests (black star), compared with the distributions of efficiency for null network models with the same number of vertices, the same number of edges, but connections have been randomly rewired. The rewiring preserves the spatial organization of the network, that is, only vertices that are adjacent in space can be physically connected by an edge.

Red box plots: distribution of the efficiency of 10000 rewired networks with the same number of vertices, the same number of edges and respecting the same spatial constraints (only vertices adjacent in space can be connected). The boundaries of the box correspond to the 25th and 75th percentile; the whiskers to the 5th and 95th percentile.

In spite of differences from one nest to the other (in part due to their different sizes), the networks made by termites are always more efficient than

Figure 2. Black stars: average topological efficiency of five nests of Cubitermes (in the abscissa).

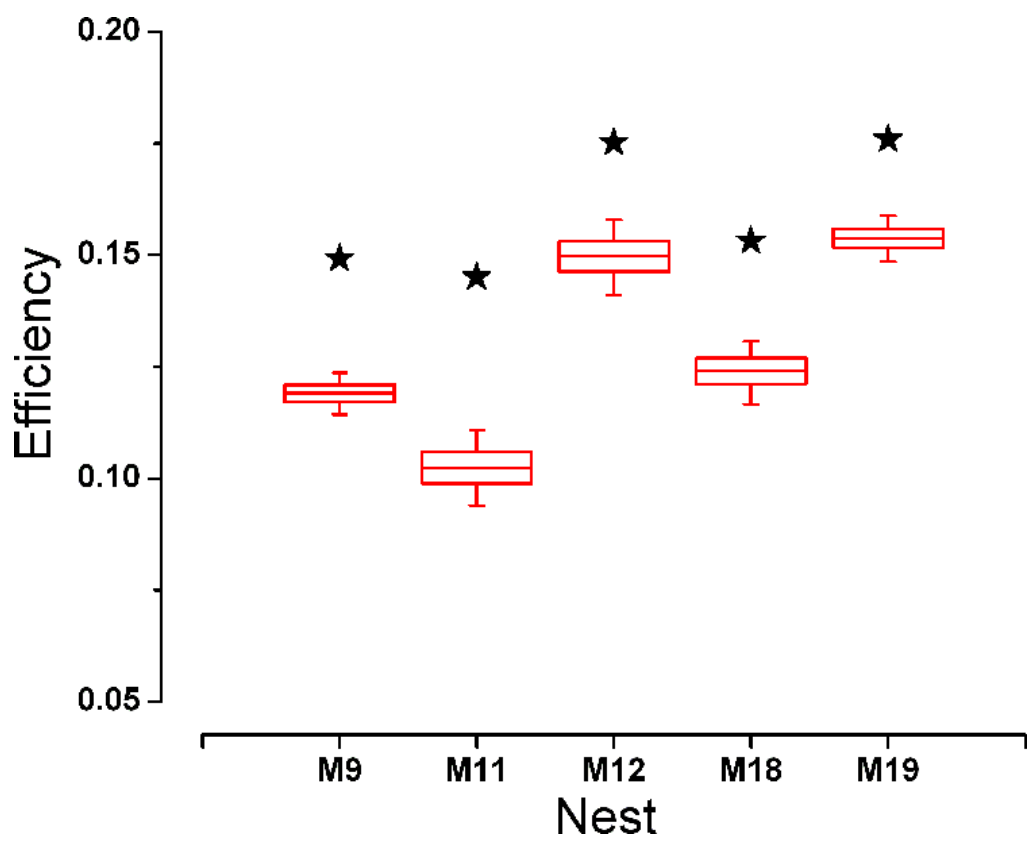


random networks with similar connectivity rules. The higher efficiency of real networks can be explained in part by the presence of long "ramps", or series of connected chambers on the vertical axis, granting fast communication from bottom to top of the nest. One of these ramps is visible in Figure 1-D, where the chambers and galleries of Figure 1-B have been flattened to improve visibility.

\section{Efficient Local Growth Rules}

From the point of view of individual insect behavior, some basic mechanisms were already highlighted in the end of the 50's by the French biologist P. P. Grassé, who introduced the concept of stigmergy (Grassé, 1959). Grassé showed that the coordination and regulation of the building activity is controlled by the growing nest structure: the local configuration of the environment and work in progress triggers particular building behaviors in the insects. The insects change their own environment as a result of their actions, and the new configuration of the environment serves as stimulus that triggers the actions of other insects. This very general mechanism explains how large numbers of insects can coordinate their respective activities (Bonabeau et al., 1999). However, this does not explain the link between perception and action of individual insects at a very small scale and harmonious growth of structures three orders of magnitude bigger.

Two broad classes of mechanisms are possible: (i) insects rely on purely local information and local rules of behavior, but these rules have been improved and refined by means of natural selection in such a way that they lead naturally to the appearance of efficient large-scale structures. The other possibility is that (ii) insects make accurate inferences about global structural properties and tune their behavior accordingly. We show in the following how an evaluation of large scale properties is not incompatible with a small perceptual range.

\section{Local Arrangement of Simple Motifs}

For layered structures similar to the Cubitermes nests, we can imagine simple stigmergic rules relying on local information only, and yet producing efficient large-scale networks.

A simple algorithm based on local decision only could be the following. Let us first consider a chain of a fixed number of vertices positioned on a horizontal straight line (layer 1). Randomly select one vertex $\mathrm{x} 1$ on the chain. Add a new vertex $\mathrm{x} 2$ on the layer 2 above $\mathrm{x} 1$ and connect it to $\mathrm{x} 1$. On the layer 2 , build a chain from $\mathrm{x} 2$ by successively adding on the right (resp. on the left) new adjacent vertices above those of the previous layer. Stop the chain when there is no vertex below in the previous layer. Repeat the process $p$ times. The resulting graph is a ladder-like tree with a vertical ramp x1, x2, .., xp (Figure 3-A). This mechanism is only local: at each step the new connections -both vertical and horizontal- are added above the existing ones in a spatial neighborhood. There is no estimation of the global organization of the structure. However, the resulting tree is more efficient than a random spanning tree of the same size. For illustration, let us consider a tree built with this rule composed of 13 layers and 8 vertices per layer. Its topological efficiency is 0.16 . By comparison, random spanning trees of an equivalent $2 \mathrm{D}$ square lattice with 8 columns and 13 rows have efficiency values around 0.121 with standard error 0.001 .

While this example is probably too simplistic to explain the formation of the complex networks observed in Cubitermes termite nests, there is some evidence that transportation efficiency can be obtained from similar local mechanisms. Valverde and collaborators (2009) created random lattice networks that matched the distribution of motifs of real Cubitermes galleries. Motifs are a local network parameter, describing the set of interconnections between small groups of 3, 4 or 5 vertices (Figure 3-B). Here, the only match for global properties was a limit on the total number 
Figure 3. A. A simple tree network obtained with no evaluation of global performance. A single vertical ramp connects all the layers (L1, L2, ..., L13). B. Possible subgraphs of four vertices for undirected graphs. C Global topological efficiency of real Cubitermes networks (dark blue) and of random spatial networks matching the frequencies of four-subgraphs found in the real graphs (yellow). In spite of the fact that the matching involves subgraphs of only four vertices, the model provides a very good approximation to the global efficiency of Cubitermes, at least for nests M12, M18 and M19. (Redrawn from Valverde et al., 2009)
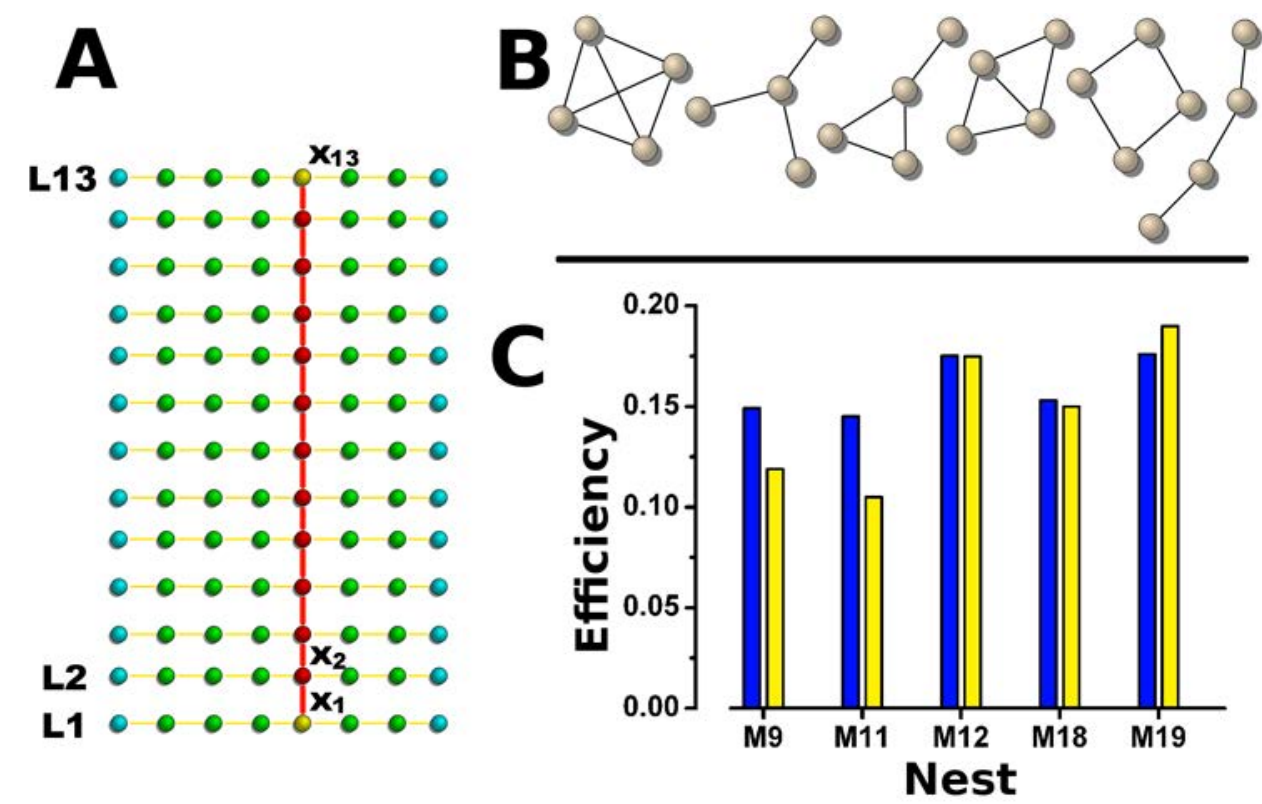

of vertices and on the dimensions of the whole lattice. Yet, the networks produced with this technique matched very well the transportation efficiency of real nests (Figure 3-C). While this was not the main purpose of their study, such results indicate that it is possible to obtain networks with similar global properties only by mixing small-scale features (the motifs) in the correct proportions, a technique that -at least in principlewould also be accessible to termites.

\section{Local Estimation of Global Properties}

Purely local mechanisms can possibly explain the formation of efficient large-scale structures. Yet, they might not allow adjusting the organization of a structure in response to environmental changes

or singular unpredictable events. The growth of a colony itself requires that the nest is continuously adapted to fulfill new needs and constraints (see also Deneubourg et al., 1986).

For these reasons, it seems reasonable that optimization of the structures is better achieved if insects have a way to evaluate the efficiency of the current solution and of improving on it based on information about some global parameters of the existing structure.

Assessing the organization and functionality of a large structure typically requires computing measures of distance, of size, of number of elements, both for the whole structure and for its parts. Clearly, insects cannot overcome the limitations imposed by their perceptual range: they cannot directly estimate the volume of the 
nest, the number of individuals in the colony, the length of the path between remote destinations, at least not when these numbers are much bigger than their own perceptual range and cognitive capacities. However, the ratios between any two such quantities can always be estimated on a local basis and may also provide cues to relevant global properties. For instance, the density of individuals inside the nest carries information about both the total number of individuals and the nest size; the traffic on a foraging trail carries information about both the number of individuals foraging on that trail and the distance of the food source ${ }^{1}$. There is large evidence that similar cues are used by insect colonies. The frequency of intersections of marked paths inside a cavity carries information about the size of the cavity and the total length of marked paths. Observations have proved that this measure is used by ants, at least in the simplest case, where one single ant explores a potential nest site. In this case, the total length of marked paths can be controlled by the ant, and the frequency of intersections gives an accurate estimate of total nest size (Mallon \& Franks, 2000). Ants of the species Leptothorax albipennis appear to regulate nest size through density, as they are able to maintain a constant ratio such that each adult worker has about $5 \mathrm{~mm}^{2}$ of floor area in the nest (Franks et al., 1992). Messor sanctus ants in laboratory conditions dig complex networks of galleries whose size is proportional to the number of individuals in the colony, suggesting that a similar mechanism is in place also for this species (Buhl et al., 2004). Similarly, Argentine ants Linepithema humile can find the shortest path from the nest to a source of food, measuring only the local concentration of pheromones or other chemicals laid by nest mates (Goss et al., 1989; Deneubourg et al., 1990). Pheromone concentration depends on the ratio between number of insects on a trail and on the length of the trail, but since the numbers of insects choosing each trail are more or less equivalent in the beginning, all the information carried by pheromones is about path length.

Can similar mechanism underlie the optimization of transportation efficiency in larger networks such as those made by Cubitermes termites?

One mechanism of network optimization based on local estimation of global properties is illustrated on a lattice model in Figure 4. Here, the global parameter to optimize is still network efficiency, and the local quantity estimated is traffic at individual edges. In terms of graphs, the amount of traffic on a vertex or edge is expressed by the betweenness centrality of the vertex or the edge. The betweenness centrality (BC) of the element $\nu \in G$, either a vertex or an edge, is defined as follows:

$B C(\nu)=\sum_{v_{i} \neq v_{j}} \frac{c_{i j}(\nu)}{c\left(v_{i}, v_{j}\right)}$

where $c i j(v)$ is the number of shortest paths from vertex $v i$ to vertex $v j$ passing through element $v$ and $c(v i, v j)$ is the total number of shortest paths from $v i$ to $v j$ (Anthonisse, 1971).

In the example of Figure 4, starting from a complete lattice, all the edges are marked with their value of betweenness centrality and the edge with lowest betweenness ${ }^{2}$ is removed from the network (unless its removal leads to disconnect the network). If the process is iterated until the network becomes a tree, the final network has an efficiency of 0.152 (and almost no variability if the to-be-removed edge is always picked among those with lowest absolute betweenness), only slightly lower than for the purely local growth mechanism tested in the previous section.

This mechanism leads to the appearance of many straight series of connections, that may be reminiscent of the vertical ramps found in the real Cubitermes nests.

Could a similar mechanism determine the optimization of termite networks? There is some evidence that young Cubitermes nests have more 
Figure 4. Network optimization based on local estimation of global properties. At each step the algorithm computes the betweenness centrality of edges and progressively removes from the network the edges with low betweenness, unless their removal triggers disconnections. Betweenness centrality can be estimated at local positions (see text), but it provides indirect information about global network parameters such as network size and path length.

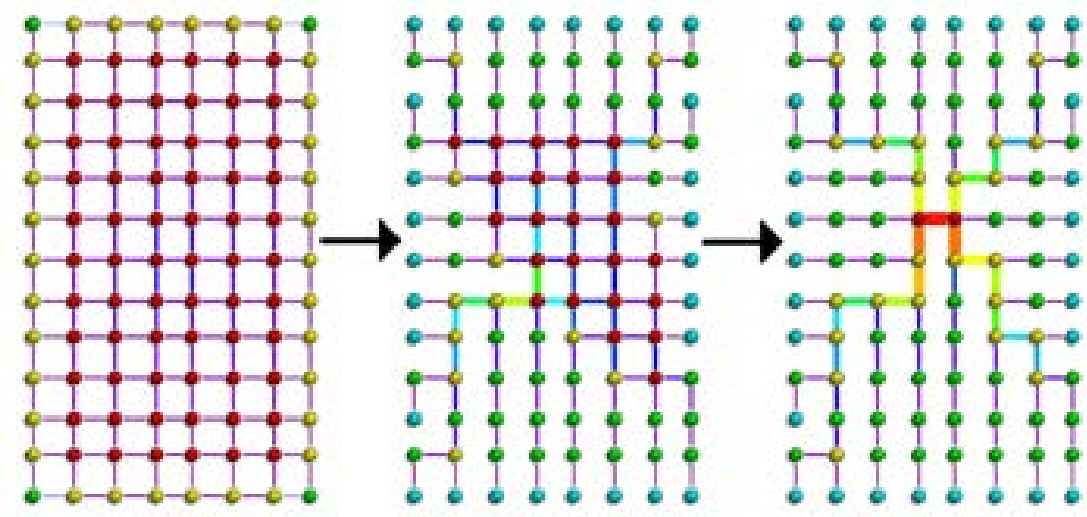

densely connected networks (i.e. networks with higher average vertex degree). This indicates that some edges are removed from the network over time. In a previous work (Perna, 2008) we used the low-betweenness removal algorithm to model Cubitermes networks. For each nest we considered the "maximal embedded graph"(MEG), the graph that we would obtain if all adjacent chambers were connected by a corridor. Starting from this nest we iterated the betweenness computation and edge removal procedure till we got a network with the same number of edges as the real termite networks (henceforth a "maximum centrality spanner").

This mechanism leads to networks much more efficient than the real ones (Perna et al., 2008). To make an example, Figure 5 represents a Cubitermes network (in B) alongside with two comparison models: a random spanner of the MEG (in A) and a maximum centrality spanner (in C). The maximum centrality spanner has a diameter (as well as other distance measures) much shorter than the other networks. It seems plausible that an implementation of the same algorithm including more noisy edge removal, different initial conditions and possibly additional constraints on the number of paths that can transit through a single node could lead to the creation of networks more similar to the real termite networks. However, in the present paper we are interested in the general mechanisms of optimization used by social insects and a detailed understanding of the exact factors shaping the form and connectivity of Cubitermes nests has little relevance for our present discussion.

We want instead to draw attention to the fact that this class of mechanisms, involving an evaluation of the global properties of a structure, or a network, is only possible if there already is a "global" structure. The structure for which global parameters are evaluated must be an already active and functional one. For this reason we argue that this mechanism of optimization is more likely to work through pruning or reshaping of an existing structure, than concurrently with its primary formation.

\section{CONCLUSION}

We have discussed two main classes of mechanisms that underlie optimization in social insect 
Figure 5. B. Flattened representation of a Cubitermes network (M11). A. Random network complying with the same spatial constraints as B (that is, only physically adjacent nodes can be connected), but with randomized connections. C. "Maximum betweenness" network obtained by iteratively removing low-betweenness edges (see the main text for a more accurate description). The node and edge colouring marks the network diameter in each of the three figures. The diameter contains 44 nodes in A, 23 in $B$ and 17 in $C$.

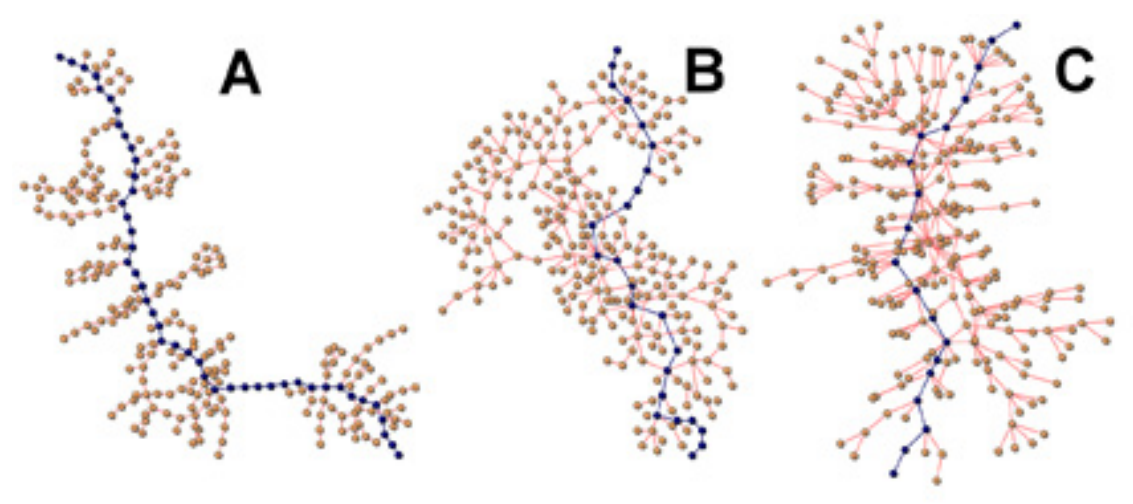

networks: purely local mechanisms, where predetermined rules of behavior lead to the arrangement of simple motifs in an efficient way, and mechanisms involving a distributed estimation of the global form and function of the structure.

Predetermined local rules of behavior can be thought of as genetically determined behavioral modules that have been optimized throughout evolution. The family of networks that can be grown through this class of mechanisms is completely specified by the local rules and their probabilistic range of application. This implies that there is no fine-tuning of parameters during the growth of the individual network or structure and in response to the current performance.

Even if the network gets close to optimal performance at some stage of its growth, this does not affect its subsequent evolution (e.g. trapping the system into a local optimum) for the simple reason that the global performance is not evaluated by this class of mechanisms.

However, purely local mechanisms do not allow for a dynamic optimization or fine tuning of the properties of a structure to fulfill unpredicted requirements, because the family of pos- sible results and their frequency of appearance is predetermined. On the contrary, mechanisms involving an estimation of global properties allow the structure to adapt to new conditions but can only work on already functional structures and are more likely to be important at later stages of network formation.

When the size of a structure is much bigger than the size of insects, insects cannot directly estimate the global properties of their network. We have discussed how some of these properties can be inferred from local properties that correspond to the ratios between two global properties.

Another mechanism that is likely to provide social insects with cues about the shape and size of a structure much bigger than themselves is the exploitation of naturally occurring environmental gradients. Temperature, humidity, irradiation and other physical and chemical quantities are not uniform throughout the environment, but may form spatio-temporal gradients at different scales. The moisture of soil increases with depth, wind speed increases with height above ground, thermal fluctuations decrease when moving deep underground. It seems plausible that these gradients act 
as cues, e.g. to indicate to the insects the depth of a tunnel, or the height of a nest.

Gradients of temperature and humidity were shown to drive digging activity in ants (Thomé, 1972; Hangartner, 1969), and air currents change the properties of macroscopic spatial structures realized by ants (Jost et al., 2007) e.g. triggering their building behavior (their probability to drop new pellets) (Bollazzi, 2007).

We can speculate that the gradient of humidity, coming from the soil and moistening the nest walls could provide Cubitermes termites with information about the current height of their nest. This would explain the fact that, in a small sample of nests, the nests originating from Savannah regions (with higher insulation and desiccation rates) are on average shorter than nests originating from shaded forest regions. However, the data currently available are not sufficient to resolve this issue.

This paper is mostly about biology: we try to understand the mechanisms of network optimization in real insect societies. We hope that our work will be a source of inspiration to computer scientists willing to explore similar mechanisms beyond the biologically plausible.

Often, the models made by biologists do not have to go back "into the jungle", they need not be functional copies of the biological system. On the contrary, bio-inspired systems are usually required to have full functionality. For this reason, we look at bio-inspired systems also as the real "ultimate test" of biological hypotheses.

\section{ACKNOWLEDGMENTS}

This work was supported by a research grant from the MESOMORPH project (French National Research Agency ANR-06-BYOS-0008). Andrea Perna would like to acknowledge John Wenzel (Ohio State University) for inspiring discussion. We are grateful to Sergi Valverde for comments to an earlier draft of this paper.

\section{REFERENCES}

Acosta, F. J., Lopez, F., \& Serrano, J. M. (1993). Branching angles of ant trunk trails as an optimization cue. Journal of Theoretical Biology, 160(3), 297-310. doi:10.1006/jtbi.1993.1020

Anthonisse, J. M. (1971). The rush in a directed graph. Technical report, Stichting matematisch centrum, Amsterdam.

Boccaletti, S., Latora, V., Moreno, Y., Chavez, M., \&Hwang, D. (2006). Complex networks: Structure and dynamics. Physics Reports - Review Section of Physics Letters, 424, 175-308.

Bollazzi, M., \& Roces, F. (2007). To build or not to build: circulating dry air organizes collective building for climate control in the leaf-cutting ant Acromyrmex ambiguus. Animal Behaviour, 74, 1349-1355. doi:10.1016/j.anbehav.2007.02.021

Bonabeau, E., Dorigo, M., \& Theraulaz, G. (1999). Swarm intelligence: From natural to artificial systems. Oxford University Press.

Buffon, G.-L. L. (1753). Histoire naturelle générale et particulière avec la description $d u$ cabinet du roi, tome IV. Imprimerie Royale, Paris. Retrieved August 15, 2010, from http://gallica. bnf. fr/ ark:/12148/bpt6k9749f

Buhl, J., Gautrais, J., Deneubourg, J. L., \& Theraulaz, G. (2004). Nest excavation in ants: Group size effects on the size and structure of tunneling networks. Naturwissenschaften, 91(12), 602-606. doi:10.1007/s00114-004-0577-x

Buhl, J., Gautrais, J., Solé, R., Kuntz, P., Valverde, S., Deneubourg, J. L., \& Théraulaz, G. (2004a). Efficiency and robustness in ant networks of galleries. The European Physical Journal B, 42, 123-129. doi:10.1140/epjb/e2004-00364-9

Buhl, J., Hicks, K., Miller, E., Persey, S., Alinvi, O., \& Sumpter, D. (2009). Shape and efficiency of wood ant foraging networks. Behavioral Ecology and Sociobiology, 63, 451-460. doi:10.1007/ s00265-008-0680-7 
Darwin, C. (1859). The origin of species by means of natural selection or the preservation of favoured races in the struggle for life. Retrieved August 15, 2010, from http://www. literature. org/ authors/ darwin-charles/the-origin-of-species-6th-edition/

Deneubourg, J. L., Aron, S., Goss, S., \& Pasteels, J. (1990). The self organizing exploratory pattern of the Argentine ant. Journal of Insect Behavior, 3, 159-168. doi:10.1007/BF01417909

Deneubourg, J. L., Aron, S., Goss, S., Pasteels, J. M., \& Duerinck, G. (1986). Random behaviour, amplification processes and number of participants: How they contribute to the foraging properties of ants. Physica D. Nonlinear Phenomena, 2,176-186. doi:10.1016/0167-2789(86)90239-3

Franks, N. R., Wilby, A., Silverman, B. W., \& Tofts, C. (1992). Self-organizing nest construction in ants: Sophisticated building by blind bulldozing. Animal Behaviour, 44, 357-375. doi:10.1016/0003-3472(92)90041-7

Goss, S., Aron, S., Deneubourg, J. L., \& Pasteels, J. (1989). Self-organized shortcuts in the Argentine ant. Naturwissenschaften, 76, 579-581. doi:10.1007/BF00462870

Grassé, P. P. (1959). La reconstruction du nid et les coordinations interindividuelles chez Bellicositermes natalensis et Cubitermes sp. La théorie de la stigmergie: essai d'interprétation du comportement des termites constructeurs. Insectes Sociaux, 6, 41-83. doi:10.1007/BF02223791

Hangartner, W.(1969). Carbon dioxide, a releaser for digging behavior in Solenopsis geminata (Hymenoptera: Formicidae). Psyche, 76, 58-67. doi:10.1155/1969/58428
Jost, C., Verret, J., Casellas, E., Gautrais, J., Challet, M., \& Lluc, J. (2007). The interplay between a self-organized process and an environmental template: Corpse clustering under the influence of air currents in ants. Journal of the Royal Society, Interface, 4(12), 107-116. doi:10.1098/ rsif.2006.0156

Latora, V., \& Marchiori, M. (2001). Efficient behavior of small-world networks. Physical Review Letters, 87(19). doi:10.1103/PhysRevLett.87.198701

Lee, S. H., Bardunias, P., \& Su, N. Y. (2007). Optimal length distribution of termite tunnel branches for efficient food search and resource transportation. Bio Systems, 90, 802-807. doi:10.1016/j. biosystems.2007.04.004

Mallon, E. B., \& Franks, N. R. (2000). Ants estimate area using Buffon's needle. Proceedings. Biological Sciences, 267, 765-770. doi:10.1098/ rspb.2000.1069

Parker, G., \& Smith, J. (1990). Optimality theory in evolutionary biology. Nature, 348, 27-33. doi: $10.1038 / 348027 \mathrm{a} 0$

Perna, A., Jost, C., Valverde, S., Gautrais, J., Theraulaz, G., \& Kuntz, P. (2008). The topological fortress of termites. Bio-Inspired Computing and Communication, LNCS, 5151, 165-173. doi:10.1007/978-3-540-92191-2_15

Perna, A., Valverde, S., Gautrais, J., Jost, C., Solé R., Kuntz, P., \& Theraulaz, G. (2008a). Topological efficiency in three-dimensional gallery networks of termite nests. Physica A Statistical Mechanics and its Applications, 387(24), 6235-6244.

Pirk, C. W. W., Hepburn, H. R., Radloff, S. E., \& Tautz, J. (2004). Honeybee combs: Construction through a liquid equilibrium process? Naturwissenschaften, 91, 350-353. doi:10.1007/s00114004-0539-3 
Solé, R. V., Bonabeau, E., Delgado, J., Fernandez, P., \& Marın, J. (2000). Pattern formation and optimization in army ant raids. Artificial Life, 6(3), 219-226. doi:10.1162/106454600568843

Thomé, G. (1972). Le nid et le comportement de construction de la fourmi Messor Ebeninus, Forel (Hymenoptera, Formicoidea). Insectes Sociaux, 19, 95-103. doi:10.1007/BF02224727

Thompson, D. W. (1992). On growth and form: The complete revised edition. Dover.

Turner, J. S. (2000). The extended organism: The physiology of animal-built structures. Cambridge, MA: Harvard University Press.

Valverde, S., Corominas-Murtra, B., Perna, A., Kuntz, P., Theraulaz, G., \& Sole, R. V. (2009). Percolation in insect nest networks: Evidence for optimal wiring. Physical Review E (Statistical. Nonlinear, and Soft Matter Physics, 79(6), 066106. doi:10.1103/PhysRevE.79.066106

\section{ENDNOTES}

1 There is an interesting analogy with the thermodynamical notion of extensive and intensive properties. Extensive properties of a system are physical quantities whose value is proportional to the size of the system or the amount of material contained in it: the volume, the mass, the number of molecules. Their value cannot be obtained sampling the system at a particular position. By contrast, intensive properties are scale invariant: they do not depend on the size of the system. These are the pressure, the density etc. The ratio between two extensive quantities is an intensive quantity that can always be estimated on a local basis.

2 Removing the edge with lowest betweenness is a simplification not completely correct in the context of "local mechanisms". A purely local mechanism could be "remove the edge with lowest betweenness among those in the neighbourhood of a randomly chosen vertex". 